\title{
Educación y pobreza: una aproximación documental a los procesos educativos en entornos de exclusión y desigualdad social en Chocó, Colombia*
}

\author{
Mirsa Chaverra Santos \\ Ministerio de Educación Nacional de Colombia, Quibdó, Colombia \\ samirita31@gmail.com
}

\section{RESUMEN}

La relación entre educación y pobreza es de suma complejidad e involucra múltiples actores, prácticas y discursos de diferente naturaleza y grados de incidencia. Por tanto, la articulación y análisis de las singularidades del contexto social son esenciales en el marco de gestión de cualquier proyecto educativo social tanto para el éxito en el proceso como para el tratamiento y disminución de la pobreza, la exclusión y la desigualdad social. Ante esto, el presente artículo tiene como objetivo general examinar la relación que existe entre educación y pobreza en algunos estudios e investigaciones publicadas durante la última década, dirigidos al análisis de los procesos educativos en el departamento del Chocó a partir de una revisión biblio- gráfica y documental. El análisis de las fuentes encontradas permitió establecer que la educación centrada en el contexto social (etnoeducación) es fundamental en el tratamiento de la pobreza, cuya incidencia se encuentra determinada por variables y singularidades del contexto mismo que deben ser analizadas e incorporadas en las políticas públicas, contenidos y proyectos educativos, con miras al reconocimiento de la diversidad, la apropiación de las dinámicas del territorio y la vinculación de la población chocoana y de sus particularidades en las dinámicas del sistema educativo.

Palabras clave: desigualdad social; educación; exclusión social; pobreza.

Cómo citar: Chaverra Santos, M. (2019). Educación y pobreza: una aproximación documental a los procesos educativos en entornos de exclusión y desigualdad social en Chocó, Colombia. Ciencias Sociales y Educación, 9(17), 145-161. https://doi.org/10.22395/csye.v9n17a7

Recibido: 15 de enero de 2020.

Aprobado: 10 de marzo de 2020. 


\section{Education and Poverty: a Look to Educational Processes in Exclusion and Social Inequality Environments. Chocó, Colombia. 2010-2019}

\section{ABSTRACT}

The relation between education and poverty is a sum of complexities and involves multiple actors, practices, and discourses of different natures and grades of incidence. That is why the articulation and analysis of theses singularities of the social contexts are essential in the framework of the management of any educational-social project both for the success in the process as for the treatment and decreasing of poverty, exclusion, and social inequality. For this, this article has as its main goal examining the relationship between education and poverty in some studies and researches published during the last decade, directed towards the analysis of educational processes in the Chocó region through a bibliographical and documentary revision. The analysis of the sources allowed the researchers to establish that the education centered on the social context (ethno-education) is fundamental for the treatment of poverty, which incidence is determined by variables and singularities of the context itself that must be analyzed and incorporated in public policy, appropriation of territory dynamics and the entailment of the Chocoan population and their particularities in the educational system dynamics.

Keywords: social inequality; education; social exclusion; poverty.

\section{Educação e pobreza: uma aproximação documental dos processos educativos em ambientes de exclusão e desigualdade social em Chocó, Colômbia}

\section{RESUMO}

A relação entre educação e pobreza é de suma complexidade e envolve múltiplos atores, práticas e discursos de diferente natureza e graus de incidência. Portanto, a articulação e análise das singularidades do contexto social são essenciais no âmbito da gestão de qualquer projeto educativo social tanto para o sucesso no processo quanto para o tratamento e diminuição da pobreza, da exclusão e da desigualdade social. Diante disso, este artigo tem o objetivo geral de examinar a relação entre educação e pobreza em alguns estudos e pesquisas publicados durante a última década, dirigidos à análise dos processos educativos no Estado do Chocó, a partir de uma revisão bibliográfica e documental. A análise das fontes encontradas permitiu estabelecer que a educação centralizada no contexto social (etnoeducação) é fundamental no tratamento da pobreza, cuja incidência é encontrada determinada por variáveis e singularidades do contexto em si que devem ser analisadas e incorporadas nas políticas públicas, nos conteúdos e projetos educacionais a fim de serem reconhecidas a diversidade, a apropriação das dinâmicas do território e a vinculação da população dessa região e de suas particularidades nas dinâmicas do sistema educacional.

Palavras-chave: desigualdade social; educação; exclusão social; pobreza. 


\section{Introducción}

A través de los años se ha concebido la educación como una manera de combatir la pobreza. Este hecho se constata en países como India, China, Singapur, Finlandia y Corea del Sur, donde la "obsesión por la educación" ha generado crecimiento económico en estos lugares (De Sousa, 2011). Sin embargo, pese a que la educación es un tema de amplio renombre en todo contexto social, en este lado del mundo la desigualdad y la exclusión social son una característica histórica y estructural que se ha mantenido y constituido en un obstáculo para erradicar la pobreza, por lo que se deduce que la educación per se no rompe este círculo vicioso (Naciones Unidas, 2019). Basta con girar la mirada hacia América Latina y el Caribe para observar la agudización de la desigualdad y la exclusión en muchos contextos y territorios, como derivación de una limitación de acceso a las oportunidades, entre ellas las educativas por limitaciones asociadas con el nivel socioeconómico de los estudiantes (Aponte, 2008).

Las nociones de "pobreza" y "educación" corresponden a constructos relacionados con supuestos e intenciones sociales que a su vez responden a los intereses de diversos grupos sociales específicos (Bazdresch, 2001). Por ello, tanto la educación como la pobreza, como categorías de estudio, entrañan una enorme complejidad en consideración de las múltiples relaciones y problemáticas subyacentes que de estas se derivan. Esta perspectiva implica el reconocimiento de las transformaciones en las dinámicas y los componentes de esta relación, así como de los factores condicionantes suscitados a partir de las singularidades del contexto.

De igual manera, estas relaciones implican la convergencia de múltiples variables de diversa naturaleza que inciden de manera determinante en la articulación de procesos educativos y de enseñanza-aprendizaje, así como en términos de la formulación de políticas para el manejo de la pobreza y la solución de las problemáticas. En este sentido, el tratamiento de fenómenos como la exclusión, la inclusión, la equidad, la inequidad, la igualdad, la desigualdad, entre otros, determina a una u otra categoría específica, así como a la relación establecida entre ellas. Por ello, la incidencia de la educación en la reducción de la pobreza dependerá, en gran medida, de la inclusión de la mayor cantidad de información relacionada con el contexto y la población, así como de su incorporación en las dinámicas, los contenidos, los procesos y las políticas educativas.

Por todo lo anterior, el presente artículo tiene como objetivo examinar la relación entre educación y pobreza en algunos estudios e investigaciones publicados durante la última década que analizan los procesos educativos en el departamento del Chocó (Colombia) a partir de una revisión bibliográfica y documental. La reflexión se formula desde la valoración de las características del 
contexto del departamento, pues en este entorno se ha evidenciado la exclusión y la desigualdad social en la población indígena y afrocolombiana: "con el mayor índice de población con necesidades básicas insatisfechas (NBI), es decir: es el departamento que presenta mayor rezago social y el más bajo nivel de desarrollo humano en todo el país" (Gobernación del Chocó, 2016, p. 16).

En el contexto colombiano sucede lo mismo. Allí se evidencian grandes disparidades en cuanto al acceso a la educación y a las oportunidades en sus diferentes territorios, lo cual se refleja en su posición en el listado de países con mayor desigualdad social. En el índice de Gini ${ }^{1}$ Colombia se ubica en las tres primeras posiciones de países desiguales en América Latina y entre las diez primeras del mundo con una valoración de 49,7, según estimaciones del Banco Mundial para el año 2017 (Banco Mundial, 2019). Dentro del contexto colombiano, especialmente el departamento ${ }^{2}$ del Chocó, las tasas de pobreza en zonas rurales superan el $62 \%$ (DNP, 2017).

Metodológicamente, para la revisión bibliográfica y documental se realizó un estudio de tipo cualitativo, teórico y descriptivo en función de la revisión de las investigaciones doctorales, los artículos científicos y los trabajos publicados en los últimos veinte años sobre la relación categórica referenciada en dicho contexto. Para la selección de la muestra documental a revisar, se recurrió a la consulta de las categorías con delimitación de búsqueda de título, tema o autor de publicaciones en algunas bases de datos académicas y repositorios de universidades nacionales e internacionales.

La tabla 1 muestra la estrategia de búsqueda implementada para el rastreo y uso de la documentación disponible sobre el tema:

Como mecanismo para el desarrollo de la consulta referenciada, se delimitaron los conceptos de educación y pobreza como nociones transversales de encuadre para la selección de los estudios enfocados en esta relación categórica, así como en las asociaciones con otras categorías. Posteriormente, se delimitó la búsqueda a referencias relacionadas con el contexto de estudio seleccionado.

Corresponde a una medida económica utilizada para calcular la desigualdad de ingresos que existe entre los ciudadanos de un territorio, generalmente de un país. Este valor se ubica entre 0 y 100; cero (0) es la máxima igualdad —todos los ciudadanos tienen los mismos ingresos - y cien (100) la máxima desigualdad —todos los ingresos los tiene un solo ciudadano- (Banco Mundial, 2019).

2 Es una división administrativa y política del territorio en tanto región geográfica, cultural y económica. Esta noción se acoge en Colombia a partir de la Constitución Política del año de 1991, donde se establece como República Unitaria dividida en treinta y dos departamentos y el distrito capital de Bogotá. A su vez, estos departamentos están constituidos por municipios que también corresponden a entidades administrativas con limites claramente definidos que pueden agrupar una o varias localidades (Federación Nacional de Departamentos [FND], 2018). 
Tabla 1. Estrategia de búsqueda

\begin{tabular}{|c|c|c|c|c|}
\hline $\begin{array}{c}\text { Bases de datos } \\
\text { consultadas }\end{array}$ & $\begin{array}{l}\text { Criterios de } \\
\text { selección }\end{array}$ & $\begin{array}{c}\text { Palabras clave o } \\
\text { descriptores }\end{array}$ & $\begin{array}{c}\text { Fecha de la } \\
\text { búsqueda }\end{array}$ & $\begin{array}{c}\text { Autores de artículos } \\
\text { hallados }\end{array}$ \\
\hline $\begin{array}{l}\text { - Alfa Omega Cloud. } \\
\text { - Bibliotechnia. } \\
\text { - Cambridge Journals. } \\
\text { - Scielo. } \\
\text { - Redalyc. } \\
\text { - Dialnet. }\end{array}$ & $\begin{array}{l}\text { - Artículos y li- } \\
\text { bros. } \\
\text { - Documentos } \\
\text { que traten te- } \\
\text { mas sobre edu- } \\
\text { cación y po- } \\
\text { breza. } \\
\text { - Documentos } \\
\text { que traten te- } \\
\text { mas sobre el } \\
\text { departamento } \\
\text { del Chocó. }\end{array}$ & $\begin{array}{l}\text { - Contexto social } \\
\text { en Chocó. } \\
\text { - Desigualdad y } \\
\text { exclusión. } \\
\text { - Singularidades } \\
\text { del contexto so- } \\
\text { cial. } \\
\text { - Relación exis- } \\
\text { tente entre edu- } \\
\text { cación y po- } \\
\text { breza. }\end{array}$ & 2017-2019 & $\begin{array}{l}\text {-Bazdresch (2001) } \\
\text {-DNP (2015) } \\
\text {-Zemelman (2008) } \\
\text {-Mineducación (2016) } \\
\text {-Schmelkes (1995) } \\
\text {-Castillo (2016) } \\
\text {-Tobón (2014) } \\
\text {-Cafam, Diócesis } \\
\text { de Quibdó, Fucla y } \\
\text { Oimusaid (2013). } \\
\text {-McLaren (2003) } \\
\text {-Arango (2008) }\end{array}$ \\
\hline
\end{tabular}

Fuente: elaboración propia.

Como justificación de este artículo, una pesquisa con el alcance y propósito indicados no es más que una respuesta a los desafíos de una realidad educativa cada vez más compleja. En este contexto, se presenta la necesidad impostergable de pensar, desde la investigación científica y la rigurosidad metodológica, la educación y la pobreza especialmente en un entorno caracterizado por la exclusión y la desigualdad social, como lo es el departamento del Chocó. Además, este contexto corresponde con la cotidianidad de muchas naciones de América Latina y el Caribe.

Igualmente, esta aproximación es una contribución académica al debate y a la reflexión sobre las carencias de la educación, entendidas como causas o consecuencias de la pobreza, lo cual apunta, en términos generales, al análisis de la relación entre ambos conceptos en consideración de las singularidades del contexto.

Asimismo, en este artículo se plantea una reflexión que resulta conveniente y oportuna si consideramos que, como bien lo han expresado Blanco y Cusato (2003), en América Latina persisten la pobreza, la desigualdad distributiva y la exclusión social. Además, es una de las regiones más inequitativas del mundo, lo que ha dificultado enormemente la constitución de sociedades más integradas.

Para el desarrollo del objetivo propuesto, este artículo presenta dos apartados. En el primero se aborda el contexto del departamento del Chocó del Pacífico colombiano para la comprensión de la relación categórica planteada y que, adicionalmente, permite encuadrar la problemática y describir las singularidades del contexto estudiado. El segundo apartado se refiere a la presentación de los 
resultados del análisis de los contenidos de las fuentes bibliográficas y documentales seleccionadas que permitieron dar cuenta de estas dinámicas en el Chocó. Finalmente, se plantean las conclusiones y recomendaciones generales basadas en algunas reflexiones para cerrar el hilo de la discusión planteada, así como esbozar posibles delimitaciones temáticas y nuevas líneas de trabajo y reflexión académica para trabajos posteriores.

\section{Una mirada al contexto del departamento del Chocó del Pacííico colombiano}

Conviene, en primer lugar, hacer un breve esbozo del contexto colombiano antes de hacer un viraje al departamento del Chocó. Colombia es un país de América del Sur con una densidad de población moderada de cuarenta y tres habitantes por $\mathrm{km}^{2}$ o, lo que es lo mismo, con una población total de 48.258.494 personas (DANE, 2018). Se destaca por estar entre los países con mayores tasas de desempleo en el mundo y es la economía número cuarenta por volumen de PIB $^{3}$. Para el año 2017, su deuda pública ascendía a 137.448 millones de euros, lo que corresponde al 49,78 \% del PIB. En Colombia se presenta una variación anual del IPC ${ }^{4}$ del 3,2 \% según la medición publicada en el segundo semestre del año 2018, con un PIB per cápita en este mismo año de $5.632 €$. Se evidencia así que los ciudadanos colombianos tienen un nivel de vida muy bajo con relación al resto de países en términos de promedio de ingresos (Banco Mundial, 2018).

Las tasas de pobreza en Colombia son altas. Tiene variaciones según la región del país y se pronuncia más en las zonas rurales. Incluso más del $55 \%$ se concentra en departamentos como La Guajira, y en Cauca y Chocó superan el 62 \% (DNP, 2017). Así las cosas, y en consonancia con lo indicado por el Banco Mundial, en Colombia la capacidad de los gobiernos locales para llevar a cabo sus funciones y desarrollar políticas públicas varía considerablemente (Mineducación, 2016). Esto incluye, por supuesto, lo relacionado con las políticas encaminadas a la gestión de procesos educativos y la reducción de la pobreza.

Toda alusión a las particularidades del contexto colombiano incluye obligatoriamente una referencia al conflicto armado interno porque este determinó el rumbo de la historia del país durante más de cinco décadas ${ }^{5}$ y hoy ha transmu-

\footnotetext{
Producto Interno Bruto. Es un indicador económico que refleja el valor monetario de todos los bienes y servicios finales producidos por un país o región en un determinado periodo de tiempo, normalmente un año (Callen, 2008).

4 Índice de Precios al Consumo. Corresponde al valor numérico que refleja las variaciones que experimentan los precios en un periodo determinado, generalmente un mes o un año (DANE, 2006).

5 Si bien la historia de Colombia está marcada por una ola de violencia desde los procesos independentistas hasta la época de la violencia bipartidista de los años cincuenta y sesenta, cronológicamente, el conflicto armado interno data desde hace un poco más de cincuenta años (Moreno, 2017).
} 
tado como consecuencia de la firma de los acuerdos de paz entre el gobierno de turno y la guerrilla de las FARC-EP ${ }^{6}$. El conflicto armado colombiano ha tenido un impacto decisivo en todos los aspectos del desarrollo: en lo económico, lo social y especialmente en la educación, dado que "coarta las posibilidades de que los niños, niñas y jóvenes asistan a la escuela, ya sea por las secuelas del traumatismo que acarrea la guerra o por la destrucción sistemática de las estructuras de la escuela" (Mosquera y Tique, 2014, p. 120). Los departamentos costeros y fronterizos, entre ellos Chocó, han sido los más afectados (Mineducación, 2016).

Ya entrando en el contexto que concierne a este artículo, el Chocó es un territorio diverso y polifacético de contrastes y contradicciones, de pueblos indígenas y comunidades negras, pero también refugio de cimarrones y libertarios (Organización Internacional para las Migraciones [OIM], 2015). Hoy en día, tan solo el 1,04\% de los colombianos viven en sus localidades. El 94,8\% de la población del departamento pertenece a grupos étnicos (afrodescendientes, indígenas y mestizos) y el 64,4 \% corresponde a personas menores de veintiséis años, lo que vislumbra su potencial de crecimiento demográfico, pese a su demostrado estancamiento social (OIM, 2015).

La riqueza y diversidad de la región se evidencian a simple vista, especialmente en sus características hidrográficas y biológicas que lo hacen uno de los lugares más lluviosos del mundo con registros promedio de hasta $12.000 \mathrm{~mm}^{3}$ anuales (Botero, 2010). Pese a ello, la violencia es una constante, con manifestaciones tanto simbólicas como estructurales. Bien lo ha expresado la OIM (2015) cuando afirma que en este territorio han quedado encapsuladas viejas conflictividades sociales, las cuales se han potenciado con la llegada de otros conflictos contemporáneos. Esto ha ocasionado impactos desproporcionados a las comunidades afrocolombianas e indígenas residentes, especialmente a los niños, niñas, adolescentes y jóvenes que se ven obligados, en muchos casos, a interrumpir sus procesos educativos, ya sea por los traumas que ocasiona la violencia o por la destrucción sistemática de las estructuras de la escuela (Mosquera y Tique, 2014).

Debe considerarse también que en este territorio se concentra un significativo número de alertas de vulneración de derechos de niños, niñas y adolescentes por cuestiones étnicas y de género. Asimismo, estos mueren violentamente o por causa de la desnutrición crónica y por carencias en la atención médica (OIM, 2015).

Finalmente, la desigualdad, según los hallazgos de la revisión de mediciones y estudios regionales (Cámara de Comercio del Chocó, 2015; DANE, 2015; Función Pública Colombia, 2019; Rodríguez y Gaviria, 2013), resulta ser uno de

Fuerzas Armadas Revolucionarias de Colombia-Ejercito Popular, transformadas en el partido político Farc como Fuerza Alternativa Revolucionaria del Común después de la firma de los Acuerdos de Paz de La Habana. 
los factores con mayor incidencia para obstaculizar el acceso a la educación en el territorio. Las diferencias en cuanto a oportunidades son irrefutables, basta observar cómo los ingresos per cápita en Bogotá son de cinco a seis veces más altos que en el departamento del Chocó. En el mismo sentido, el porcentaje de la población con NBI en la ciudad capital corresponde a un $20 \%$, mientras que en el Chocó este valor es superior al 65 \% (Mineducación, 2016). Asimismo, la tasa de analfabetismo excede el $20 \%$ en este departamento, mientras que en Bogotá y en otros territorios puede alcanzar tan solo el 6 \% (Mineducación, 2016).

\section{Una aproximación a la relación entre educación y pobreza en el departamento del Chocó}

El desarrollo de la estrategia metodológica descrita en la introducción de este artículo permitió establecer, en primera instancia, dos líneas de pensamiento: la primera se centra en establecer cifras y mediciones para determinar la pobreza y la educación; esta relación es tratada tangencialmente en algunos trabajos que aluden a otros aspectos de las ciencias de la educación. En este sentido, son numerosas las mediciones demográficas y econométricas desarrolladas en este contexto, donde la educación es considerada como un rubro de incidencia presupuestal, una variable económica y no como un proceso complejo donde intervienen múltiples actores y se derivan variadas implicaciones para el desarrollo social.

Por otra parte, está la línea de pensamiento que tiene en cuenta las singularidades del contexto social más allá de lo propuesto por el Estado y otros entes en términos de la estandarización de ambos conceptos. Este discurso se apega a metodologías de investigación concentradas en métodos de corte social y tiene como principal concepto la etnoeducación.

A partir de estas dos líneas, la revisión posibilitó la identificación de algunos enfoques aplicados en el desarrollo de estos trabajos, así como de categorías, aspectos, prácticas, discursos y dimensiones que subyacen en el establecimiento de la relación entre educación y pobreza. La mayoría de los estudios analizados correspondieron a pesquisas con enfoques cualitativos de corte descriptivo mediante la aplicación de distintos paradigmas de investigación, entre ellos interpretativos, de pensamiento crítico, constructivismo y sociocríticos. Ahora bien, también se encuentran análisis mixtos que incluyen componentes demográficos y econométricos para la educación y el tratamiento de la pobreza. Hechas estas consideraciones generales sobre las características de la muestra de investigación y trabajos seleccionados para el análisis de la relación, se presentan a continuación las líneas de trabajo y las principales discusiones presentadas en estos desarrollos. 


\section{Línea de pensamiento de la relación entre educación y pobreza basada en la medición}

Una primera mención de obligatoria referencia es el trabajo de Bazdresch (2001), que estudia la relación entre la educación y la pobreza con importantes elementos para el debate, pues cuenta con una revisión metodológica de las teorías o marcos de referencia que han permitido la interpretación del papel de la educación en términos de su correspondencia con categorías conceptuales como la pobreza, la igualdad, la equidad y la marginalidad. Según el autor, estas categorías son frecuentemente utilizadas por el Estado y los gobiernos en función de la legitimación, significación y organización de la política educativa y de sus estrategias (Bazdresch, 2001). Esta perspectiva cuestionada por Bazdresch (2001) constituye un aspecto funcional de cómo el Estado le ha dado manejo a la pobreza y a la educación sin tener en cuenta las singularidades de cada contexto.

Por otro lado, debe considerarse que cada uno de estos constructos teóricos disponen de rejillas específicas de conceptos y enfoques que, a su vez, determinan el manejo de las variables en las políticas públicas de educación, reducción de la pobreza y en la manera en que se formulan sus relaciones. Asimismo, inciden en los criterios de priorización en el tratamiento de las problemáticas y sus efectos, lo que depende en muchos contextos de América Latina y el Caribe de los intereses de grupos particulares, así como de la implementación de algunos modelos de desarrollo que se enfocan generalmente al crecimiento económico.

Aunque el estudio de Bazdresch se centra en el análisis situacional de México, el autor presenta una completa revisión de los componentes teóricos que conviene tener en cuenta como antesala de la revisión de los trabajos que estudian el contexto chocoano. Bazdresch (2001) retoma las reflexiones y enfoques de Schmelkes en cuanto al peso e implicaciones que se derivan de la aceptación de la imposibilidad de tratar la pobreza mediante la educación.

Para el caso del departamento del Chocó, esta línea de pensamiento tiene plena validez. Considérese en este punto de la discusión que, pese a la implementación de diversas iniciativas y proyectos educativos, el índice de pobreza sigue rondando el $60 \%$, lo que significa que un estudiante chocoano, para solventar sus necesidades, gasta lo mismo que un estudiante residente en la ciudad de Bogotá, la capital de la república (DNP, 2017). En el mismo sentido, nótese cómo aquellos departamentos y municipios, que tradicionalmente se han caracterizado por asignar más fondos como mecanismo de apoyo para los estudiantes, no están viendo mejoras sustanciales en la calidad y la cobertura educativa (Mineducación, 2016).

Hallazgos de este tipo remiten a otro debate de actualidad académica que se ocupa de la crisis del modelo de desarrollo neoliberal y su impacto en 
los procesos educativos. En este sentido, resulta ilustrativo mencionar los trabajos de Zemelman (2008) en los que se ha considerado que en el mundo contemporáneo convergen ciertas problemáticas que no son más que derivaciones de los apuros que enfrenta el dechado tradicional. En este sentido, este autor ha circunscrito sus enfoques y perspectivas al desenvolvimiento de simples lógicas econométricas - diseñadas para la competencia y el libre mercadoimplementadas en el último siglo y que hoy muestran contundentes señales de fracaso (Zemelman, 2008).

En este orden de ideas, el fracaso está asociado por algunos autores, entre ellos Zemelman (2008), con la reconocida prelación que este modelo le concede a la dimensión económica del desarrollo y, por consiguiente, a la educación. En muchos casos relega la atención de los factores sociales, culturales y ambientales, tan determinantes en una sociedad cada vez más desigual, donde ciertos grupos poblacionales mayoritarios continúan siendo segregados en contraposición con la entronización de otros que, aunque son minoritarios, detentan mayor poder económico y político. En el ámbito educativo esto se ve reflejado, según palabras del mismo autor, en la reducción de la inteligencia a la llana competencia y de los procesos educativos a simples gestiones de orden administrativo (Zemelman, 2008).

\section{Línea de pensamiento de la relación entre educación y pobreza basada en la etnoeducación}

En esta línea se tiene en cuenta también a Bazdresch (2001), quien ha llamado reiterativamente la atención sobre la necesidad de analizar las carencias y la efectiva capacidad de la educación como herramienta de construcción de una sociedad justa. Asimismo, según Schmelkes (1995), resulta menester validar si los modelos educativos utilizados y las dinámicas de establecimiento de procesos de enseñanza-aprendizaje redundan efectivamente en la formación de individuos autónomos, creativos y participativos, con herramientas adecuadas para el mejoramiento del nivel de vida de la colectividad social. Así las cosas, en palabras de la autora, si bien no se puede demostrar que la educación "saca" a los pobres de la pobreza, tampoco es posible afirmar que se pueda prescindir de esta en el combate contra la pobreza (Schmelkes, 1995).

Además de las teorías, las investigaciones también han incluido en sus líneas de trabajo y discusión los enfoques, las prácticas y los discursos desarrollados en ciertos contextos con poblaciones vulnerables. Así las cosas, y atendiendo de nuevo el contexto colombiano, específicamente los procesos educativos en el departamento del Chocó, se encuentran distintas aproximaciones y publicaciones que analizan los alcances y la incidencia de la educación en el desarrollo social de un territorio afectado tradicionalmente por la pobreza, la desigualdad y la exclusión social. 
En esta línea de estudio y revisión se encuentra el trabajo publicado por Castillo (2016), que analiza el enfoque etnoeducativo afrocolombiano y de las pedagogías de la dignificación en los procesos educativos de la región Pacífica de Colombia. La autora advierte en la etnoeducación una herramienta útil para el logro del reconocimiento y la vinculación de la población chocoana y de sus particularidades en las dinámicas del sistema educativo, tradicionalmente abocado a los intereses de otros grupos humanos que ni siquiera habitan en el territorio, lo que en cierta medida constituye una imposición de una estructura mayor — sistema educativo—ideológica, epistemológica, económica y políticamente direccionada.

La etnoeducación logra incorporar las singularidades del contexto en el proceso educativo al connotar varios sentidos, entre ellos el político, cultural, territorial, intercultural e identitario en torno a la pertenencia étnica (Castillo, 2016). Bajo esta perspectiva, se asumen las problemáticas y características del contexto como elementos constitutivos de la realidad que encarnan importantes desafíos: la desigualdad, la exclusión social y la pobreza en el departamento.

Es necesario indicar que, como consecuencia de la complejidad implícita en estos retos, el proyecto etnoeducativo para la población afrocolombiana e indígena debe sortear distintos desafíos e importantes transformaciones. Asimismo, demanda nuevas interpretaciones en términos de la perspectiva de las pedagogías de la dignificación y las afroreparaciones (Castillo, 2016).

Otro autor que resalta la etnoeducación, esta vez relacionada con la pobreza, es Tobón (2014). Este autor la define como un proceso de socialización y formación que direcciona el etnodesarrollo, palabra clave para separar el desarrollo del discurso funcional y encauzarlo hacia uno acorde con las necesidades del contexto social. La etnoeducación se fundamenta en "su cultura, reconociendo a la persona y a la colectividad como sujeto de afirmación y cambio, en interacción con otras culturas" (Tobón, 2014, p. 202).

Con base en lo anterior, cualquier empresa de destino pedagógico debe incluir en la planeación y en el desarrollo de sus componentes las condiciones que rodean la vida de la población objetivo que, en el caso del Chocó, han sido transformadas dramáticamente por el conflicto armado colombiano debido a la pervivencia de economías ilegales, la presencia de actores armados, el reclutamiento de menores para la guerra, el abandono del Estado y el desplazamiento forzado que aqueja a muchos de los territorios colectivos de comunidades negras e indígenas.

Por ello, retomamos los planteamientos de Schmelkes (1995) y Bazdresch (2001) en la línea de lo anteriormente mencionado. En este sentido, debe anotarse que para la gestión de cualquier proyecto de orden educativo con efectiva 
influencia social, resulta imperativo incorporar en el análisis la mayor cantidad de datos y variables que permitan orientar las acciones y políticas gubernamentales en función de las problemáticas específicas del contexto y del tratamiento de las variables de mayor incidencia. Por un lado, así se permite el desarrollo de modelos educativos afines a las condiciones reales de existencia para toda la población y, por el otro, se propicia el tratamiento de la pobreza, la exclusión y la desigualdad social (Schmelkes, 1995).

Otro referente de investigación que desarrolla esta misma línea de pensamiento lo constituye el proyecto sobre la calidad de vida de la infancia chocoana liderado por Usaid. Este incluye una completa caracterización de la población y el territorio como conditio sine qua non para la implementación y desarrollo de las estrategias del programa. En este sentido, se direcciona a la atención integral de la primera infancia en el departamento del Chocó, en el área de educación inicial, como un esfuerzo de orden pedagógico con miras a la incorporación y análisis de las necesidades, inquietudes e intereses de los niños y las niñas de las etnias afro, indígenas y mestizas. Todo ello está en función de la potenciación de sus habilidades, competencias y destrezas mediante la apropiación de los saberes culturales y las herramientas pedagógicas y didácticas (Cafam, Diócesis de Quibdó, Fucla y Oimusaid, 2013).

En esta misma línea de trabajo convergente, basado en el reconocimiento de las singularidades del contexto y de la población, se halla la investigación desarrollada por la OIM y la Agencia de Estados Unidos para el Desarrollo Internacional (Usaid), enfocado en la construcción de una paz estable, duradera y sensible para los niños, niñas, adolescentes y jóvenes como un proceso de reflexión sobre el significado de la construcción de la paz. En este desarrollo se presenta un análisis omnicomprensivo que incluye los actores, prácticas y discursos relacionados con una efectiva construcción social de la paz, donde la educación resulta ser una herramienta de gran valor. Sin embargo, como bien lo indica la OIM, es insuficiente en comparación con los desafíos y problemáticas del entorno, por lo que resulta necesario integrar al análisis otros elementos como la salud y la identidad, que están íntimamente ligados con la educación y con el tratamiento efectivo de la pobreza, la exclusión y la desigualdad social.

El estudio incluyó el análisis de datos de entrevistas de estudiantes de la región, lo que permitió a los autores identificar la percepción local sobre la educación como acto cotidiano, es decir, sobre el hecho mismo de estudiar que no es percibido en la región chocoana como un bien capitalizable, sino como algo que irrumpe con lo que habitualmente se hace (OIM, 2015). Esto resulta determinante en términos de la convergencia de los intereses personales, los 
objetivos del proceso educativo y las necesidades del contexto, como se analiza posteriormente a la luz de los trabajos de McLaren (2003). A partir de tales hallazgos resulta conveniente examinar la valoración social de la educación, así como validar la pertinencia del sistema educativo en muchos aspectos y según el contexto.

Esta valoración y pertinencia también es importante para efectos del tratamiento de fenómenos como la inclusión y la sostenibilidad, relacionados con la pobreza y en los que incide notoriamente la educación. Por ello, se percibe como altamente necesario implementar un enfoque diferencial en el sector educativo que posibilite la promoción de educación étnica e intercultural, como mecanismo de reconocimiento y apropiación de las singularidades del contexto. En el territorio, dadas las características del contexto, la educación se combina con la economía, la reconciliación y la democracia. En este sentido, trasciende las aulas de clase para instaurarse en las dinámicas de construcción de paz. Asimismo, llega a cárceles y procesos judiciales como un tipo de demanda de justicia social.

\section{Otras líneas de pensamiento centradas en la diversidad en el aprendizaje}

El reconocimiento de la diversidad y la apropiación de las dinámicas del territorio ha sido una tesis recurrente en otras pesquisas. Muestra de ello es la investigación de corte antropológica realizada por Arango (2008), que se dedica a la exploración de los principales espacios de educación musical en Quibdó (capital del departamento del Chocó) y a analizar los escenarios emergentes de aprendizaje. La autora enfatiza las complejidades que se evidencian en el proceso de enseñanza y aprendizaje en el marco del establecimiento de relaciones de poder en los mecanismos de intercambio de saberes entre unos sectores "dominantes" y otros "subalternos" (Arango, 2008).

En la tesis de Arango (2008) sobresale la idea de que las comunidades de práctica se consolidan en el marco del desarrollo de contextos de micropolítica y en medio de discursos hegemónicos que implican imposición de formas, cuestionamientos, obstáculos y vetas para el desarrollo, bien sea de forma explícita o enmascarada. La tesis de la autora remite a los estudios escolares de McLaren sobre el fracaso escolar y la deserción como derivación de la exclusión social de subclases de jóvenes negros en condición de subordinación y marginación. Según McLaren (2003), esta dinámica de relación entre las subclases y la posición de cada una puede variar en cada entorno educativo, según las condiciones demográficas y las características de cada contexto. Adicionalmente, a una condición de exclusión se le pueden yuxtaponer otras causas, como el hecho de pertenecer a una minoría, ser inmigrante o ser mujer. Todos estos factores 
son determinantes en la manera en que el estudiante se percibe a sí mismo y asume las dinámicas del proceso educativo.

Las reflexiones de McLaren, pese a derivarse del estudio de otros contextos, permiten valorar las implicaciones de estas particularidades en la construcción de la identidad, relacionada íntimamente con la construcción de la noción de éxito en los educandos, la posición personal y la actitud frente a la pobreza. Asimismo, las condiciones económicas o la pertenencia a determinada clase, bien sea la de propietarios de los medios de producción o como miembros de la clase trabajadora, se asocia con la estratificación y la elección vocacional. De este modo, para los estudiantes pertenecientes a la clase trabajadora, por ejemplo, se percibe la concepción de no existencia de garantías para que un trabajo determinado pueda permitir, en un momento dado, el mejoramiento de la calidad de vida o, en palabras de McLaren (2003), "un buen trabajo o que la vida adulta será de alguna manera menos dura o conflictiva" (p. 306).

En esta dinámica incide la posición de la familia y de la comunidad en lo referente a la determinación de la edad en la que los hijos o los miembros menores del grupo deben ingresar al mercado laboral como complemento al sustento y apoyo para la resolución de necesidades del grupo familiar. Todas estas circunstancias determinan tangencialmente el éxito en el proceso educativo por parte de los estudiantes, especialmente por su impacto en la percepción y la actitud frente al proceso y el logro de las metas y objetivos proyectados.

Por otro lado, las relaciones citadas anteriormente se establecen en el marco del encuentro de posiciones contrarias que, como tales, resultan de las percepciones, modelos y proyecciones de los estudiantes pertenecientes a cada subclase. Asimismo, estas son las vías y herramientas que consideran válidas para el logro de sus propósitos o las maneras de llegar al éxito que, finalmente, se derivan de las visiones de cada subclase. En este orden de ideas, la dinámica escolar se muestra como un encuentro hostil entre estas visiones, donde la escuela puede oficiar en función de la reproducción mecánica de las divisiones sociales de cada clase social, y como espacio de reproducción de la estratificación laboral y diferenciación ocupacional.

Debe considerarse que las conductas y el rendimiento en el salón de clase de los estudiantes marginados y excluidos por bajos recursos, diferencias étnicas, pertenencia a minorías o a grupos vulnerables, están determinados en cierta medida por la historia como grupo social, es decir, como grupos oprimidos y marginados por sus prácticas culturales, sociales y su cotidianidad (McLaren, 2003). 


\section{Conclusiones}

Según lo anteriormente expuesto, es posible concretar lo planteado en algunas consideraciones finales que ofician como conclusiones y recomendaciones generales a partir de los hallazgos presentados.

El rastreo documental derivó en estudios teóricos y metodológicos a nivel nacional e internacional. De esta manera, se revela, por un lado, que la relación entre educación y pobreza se puede medir cuantitativamente, pero no refleja ni se anticipa a las condiciones sociales y culturales de las poblaciones excluidas y marginadas. Al medirse, ambos conceptos toman la forma de indicadores que le sirven al Estado y demás entes asociados para establecer una regulación del presupuesto y una estandarización a través de pruebas con un contexto general, mas no particular. Por otra parte, se encuentra otra línea de pensamiento más acorde y propia de comunidades afros e indígenas, donde la relación entre educación y pobreza no se ajusta a parámetros cuantitativos, sino al reconocimiento de la cultura y la diversidad. Allí la pobreza, si bien no se diluye, se reconfigura hacia el reconocimiento propio de estas comunidades con independencia de las mediciones del Estado.

Si bien es cierto que resulta necesario el tratamiento de la educación como derecho constitucional, es igualmente cierto que la gestión cotidiana de este asunto va más allá de un reconocimiento jurídico. Por tanto, hablar de educación implica trascender la simple alusión de derecho que se reconoce en un texto constitucional para dimensionarla como un proceso de notable complejidad, donde convergen distintos actores, asuntos, enfoques y perspectivas (etnoeducación).

Por otro lado, el tratamiento de la pobreza en las sociedades contemporáneas implica un tratamiento multidimensional y omnicomprensivo que se sustenta en el reconocimiento de la complejidad implícita en las prácticas y discursos que subyacen en los procesos educativos y en la sociedad en general. Allí la educación es una herramienta que, si bien resulta útil, podría no ser determinante si no se encuentra acompañada de políticas públicas y modelos educativos provechosos en el tratamiento de las problemáticas y la inclusión de las características de un contexto determinado.

La educación opera como una herramienta para la configuración del sujeto o, en palabras de Zemelman (2015), como un reconfigurador del sujeto político e intelectual. Teniendo en cuenta la crisis de los modelos de desarrollo a los que asiste la sociedad contemporánea, implican emprender el camino por la recuperación del pensamiento, esto es, recuperar la capacidad de reconocer sus circunstancias y potencializar sus capacidades en función del desarrollo personal y social a partir de la educación, lo que puede incidir en la solución de problemáticas sociales como la pobreza, la exclusión y la desigualdad social. 


\section{Referencias}

Aponte, E. (2008). Desigualdad, inclusión y equidad en la educación superior en América Latina y el Caribe: tendencias y escenarios alternativos en el horizonte 2021. En A. Gazzola, y A. Didriksson (eds.), Tendencias de la educación superior en América Latina y el Caribe (pp. 113-154). Unesco/ Iesalc.

Arango, A. (2008). Espacios de okeducación musical en Quibdó (Chocó-Colombia). Revista Colombiana de Antropología, 44(1), 157-189. http://www.redalyc.org/pdf/1050/105012924006.pdf

Banco Mundial. (2018). Datos Colombia. https://datos.bancomundial.org/pais/colombia

Banco Mundial. (2019). Índice de Gini. https://datos.bancomundial.org/indicador/SI.POV.GINI?view=map

Bazdresch, M. (2001). Pobreza, Desigualdad Social y Ciudadanía. Los Límites de Las Políticas Sociales. http://feae.eu/wp-content/uploads/2017/09/CLACSO-POBREZA-Y-EDUCACIÓN.pdf

Blanco, R., y Cusato, S. (2003). Desigualdades educativas en América Latina: todos somos responsables. http://www.red-ler.org/desigualdades_educativas_america_latina.pdf

Botero, C. (2010). El Chocó biogeográfico un tesoro de la naturaleza. Gestiópolis. http://www.gestiopolis. com/choco-biogeografico-tesoro-naturaleza/\# autores

Cafam, Diócesis de Quibdó, Fucla y Oimusaid. (2013). Guía pedagógica con enfoque etno educativo para la atención integral de la primera infancia en el choco.

Callen, T. (2008). ¿Qué es el producto interno bruto? Finanzas \& Desarrollo, 45(4), 48-49. https://www. imf.org/external/pubs/ft/fandd/spa/2008/12/pdf/basics.pdf

Cámara de Comercio del Chocó. (2015). Una mirada a la dinámica económica del chocó durante el año 2015 desde la cámara de comercio. https://camarachoco.org.co/sites/default/files/adjuntos/estudio_economico_ccch-2015.pdf

Castillo, E. (2016). Etnoeducación afropacífica y pedagogías de la dignificación. Revista Colombiana De Educación, 1(71), 343-360. https://doi.org/10.17227/01203916.71rce343.360

DANE. (2006). Índice de precios al consumidor. https://www.dane.gov.co/files/faqs/faq_ipc.pdf

DANE. (2015). Chocó-Quibdó. Informe de coyuntura económica regional. de http://www.banrep.gov. co/sites/default/files/publicaciones/archivos/icer_choco_2014.pdf

DANE. (2018). Censo poblacional Colombia 2018 ¿Cuántos somos? https://www.dane.gov.co/index. php/estadisticas-por-tema/demografia-y-poblacion/censo-nacional-de-poblacion-y-vivenda-2018/ cuantos-somos

Departamento Nacional de Planeación, DNP. (2017). Bases del Plan Nacional de Desarrollo 2014-2018. https://acp.com.co/web2017/es/leyes/200-bases-plan-nacional-de-desarrollo-2014-2018-2/file

De Sousa, M. (2011). Educación para combatir la pobreza. Sapiens. Revista Universitaria de Investigación, 12(2), 1-2. https://www.redalyc.org/pdf/410/41030368001.pdf

FND. (2018). Federación Nacional de Departamentos de Colombia. https://fnd.org.co/

Función Pública Colombia. (2019). Chocó: Ficha de caracterización departamental. https://www.funcionpublica.gov.co/documents/418537/1205912/2017-04-28_Choco_depto.pdf/4e6aaf24-b1d9-4a978854-6ee103dfbd7a 
Gobernación del Chocó. (2016). Plan de desarrollo departamental del Chocó 2016-2019. https://choco. micolombiadigital.gov.co/sites/choco/content/files/000023/1103_pdd20162019.pdf

McLaren, P. (2003). La vida en las escuelas. Una introducción a la pedagogía crítica en los fundamentos de la educación. Siglo XXI Editores S.A. https://www.uaeh.edu.mx/profesorado_honorario_visitante/ peter_mclaren/presentaciones/LA\%20VIDA\%20EN\%20LAS\%20ESCUELAS.pdf

Mineducación. (2016). Revisión de políticas nacionales de educación: La educación en Colombia. OECD. https://www.mineducacion.gov.co/1759/articles-356787_recurso_1.pdf

Moreno, E. (2017). Educación, conflicto y posconflicto en Colombia. Revista Diálogos de Saberes, (46), 125-142. https://doi.org/10.18041/0124-0021/dialogos.46.2017.2578

Mosquera, C. y Tique, J. (2014). Voces desde la escuela de Bojayá en medio del conflicto armado: construcción de su memoria colectiva. Revista de la Universidad de La Salle, (63), 117-134. https:// ciencia.lasalle.edu.co/ruls/vol2014/iss63/8/

Naciones Unidas. (2019). Panorama Social de América Latina. https://repositorio.cepal.org/bitstream/ handle/11362/44969/5/S1901133_es.pdf

Organización Internacional para las Migraciones, OIM. (2015). Una paz estable, duradera y sensible a niños, niñas adolescentes y jóvenes (1.a ed.). Organización Internacional para las Migraciones (OIM).

Rodríguez, A. y Gaviria, M. (2013). Las comunidades indígenas y afrocolombianas del Chocó promueven su seguridad alimentaria y nutricional. (Informe). Fondo Español para el logro de los objetivos del milenio (F-ODM). https://www.unicef.org/evaldatabase/files/Colombia_2013-004_INFORME_ FINAL_EVALUACION_PC_CHOCO.pdf

Schmelkes, S. (1995). Hacia una mejor calidad de nuestras escuelas. Secretaría de Educación Pública-SEP.

Tobón, C. G. (2014). Reflexiones sobre lengua, etnia y educación. Siglo del Hombre.

Zemelman, H. (2008). La formación de docentes como sujetos protagónicos en los procesos educativos. Sociologando. http://sociologandoudea.blogspot.com/2015/06/la-formacion-de-docentes-comosujetos.html

Zemelman, H. (2015). Pensamiento y construcción de conocimiento histórico, una exigencia para el hacer futuro. El Ágora USB, 15(2), 343-362. https://doi.org/10.21500/16578031.1618 